\title{
Comparative Osteointegration Testing of Titanium Implants in Relation to Gender After One Month of Implantation in Rabbit Femur
}

\author{
Teodora MARCU ${ }^{1}$, Adrian $\mathrm{GAL}^{2}$, Cristian MARTONOS², Vasile RUS², Aurel DAMIAN²*, Viorel MICLĂUȘ ${ }^{2}$, \\ Augustin T. MIHAI ${ }^{3}$ \\ ${ }_{1}^{1}$ „Dunărea de jos” Universtity of Galați, Faculty of Medicine and Pharmacy, Department of Dental Medicine \\ ${ }^{2}$ University of Agricultural Sciences and Veterinary Medicine of Cluj-Napoca, Faculty of Veterinary \\ Medicine, Department of Preclinical Sciences \\ ${ }^{3}$ University of Medicine and Pharmacy „Carol Davila” Bucharest, Faculty of Dental Medicine \\ *corresponding author: damian56aurel@yahoo.com
}

Bulletin UASVM Veterinary Medicine 77(2)/2020

Print ISSN 1843-5270; Electronic ISSN 1843-5378

doi:10.15835/buasvmen-vm:2020.0035

\begin{abstract}
The biologic material used in this study is represented by 6 rabbits, 3 males and 3 females, aged 11 months and having an average weight of $4 \mathrm{~kg}$. After general anaesthesia was performed, $2 \mathrm{~mm}$ diameter titanium implants were surgically inserted in the femur. Postoperative care of the animals included daily observation. After 4 weeks the animals were euthanized according to the guidelines. The femurs were collected along with the implants and processed for histological examination. The samples were placed in Stieve mixture for fixation, dehydrated with ethylic alcohol, clarified with 1-Butanol, and followed by paraffin embedding. 5 micrometers sections were cut using a microtome, and then stained using the Goldner trichrome staining method and were examined using an Olympus BX41 microscope with an attached digital camera for image capturing. In all animals, the implant was well tolerated by the bone and soft tissue implantation bed. The osteointegration process took place through bone formation around the implant, with variations in thickness and structure throughout the bone-implant interface. Except for the accelerated speed of osteointegration steps, observed in males, the osteointegration process follows certain stages and no gender related differences. The rapid progress of osteointegration in males resulted in a faster process in this gender compared to females. This aspect is clinically relevant for the appropriate evaluation of waiting periods from implant placement to prosthetic mounting, which according to this study is different in males and females.
\end{abstract}

Keywords: implants, osteointegration, rabbit femur, titanium,

\section{Introduction}

Mesa et al. (2008) concluded that males are less prone to primary implant failure than females, the risk being 1.54 times higher for the latter. The authors state that the smaller failure rate in males is related to a difference in bone mass between males and females. Similar results have been reported by other authors in a study conducted for the evaluation of stability of Brånemark implants by resonance frequency analysis (Ostman et al.,
2006). Different authors found better primary stability of implants in the posterior maxilla in males (Tricio et al., 1995), but no differences between genders throughout the osteointegration process (Haas et al., 1995). Other researchers state that the primary mechanical stability seen at endosseous dental implant placement gradually gives way to biologic stability after a few weeks of implant placement (Raghavendra et al., 2005). 
A meta-analysis on long-term implant survival rate was conducted by Chrcanovic et al. (2015). It included 91 studies reporting on 27203 dental implants inserted in male patients and 25154 in female patients. The authors found 1185 failures in males (4.36\%) and 1039 (4.13\%) in females. The results suggested that survival rate failure for implant placement is 1.21 times higher in males.

Although success and survival rates of osteointegrated dental implants have been studied extensively throughout the years, there is still a lot unknown about the gender influence on the results of endosseous dental implants therapy.

Considering that the ability to predict postoperative outcome is essential for implant therapy management, knowledge of several details about the patients will allow the surgeon to develop the optimal treatment plan with the best outcome (Akca et al., 2007).

As dental implants survival rate is linked to a large number of both objective and subjective factors, its evaluation will not render information on each particular factor, but rather on the cumulative effects. There is few and incomplete data found in literature regarding gender related osteointegration process evaluated by histological techniques (Pearce et al., 2007; Wancket, 2015). In view of the fact that this study aims to investigate this aspect on laboratory animals, rabbits are the recommended experimental animal model.

\section{Material and Methods}

The biologic material used in this study is represented by 6 rabbits, 3 males and 3 females, aged 11 months and having an average weight of $4 \mathrm{~kg}$. General anaesthesia was performed by intramuscular administration of $5 \mathrm{mg} / \mathrm{kg}$ xylazine and $40 \mathrm{mg} / \mathrm{kg}$ ketamine, followed by the placement of an auricular catheter for intra-operative fluid management $(5 \mathrm{mg} / \mathrm{kg})$. Surgical exposure of the femur was performed. $2 \mathrm{~mm}$ diameter self-tapping titanium implants were placed after the drilling of $1.8 \mathrm{~mm}$ diameter transcortical holes. Sutures were performed for the surgical closure of the muscular layers and the skin. Postoperative, the rabbits were administered $20 \mathrm{mg} / \mathrm{kg}$ enrofloxacin (Enroxil 5\%, KRKA), subcutaneously, daily, for 5 days and $1 \mathrm{mg} / \mathrm{kg}$ meloxicam (Metacam, Boehringer Ingelheim), subcutaneously, daily for 3 days. 4 weeks after implantation, euthanasia was performed, and the femurs were harvested along with the implants and processed for histological examination. The samples were fixed in Stieve mixture for 3 days (Gabe, 1968,), decalcifying was performed using trichloracetic acid, followed by paraffin embedding, 5 micrometers sections using a microtome and Goldner's trichrome staining technique (Gruber, 1992; Gruber, Ingram, 2003; Bancroft, Layton, 2019). Sample examination was performed using an Olympus BX41 microscope with an Olympus E-330 digital camera attached for image capturing. The images were processed using Adobe Photoshop 2020.

\section{Results and discussions}

In males, four weeks after titanium implant placement in the femoral diaphysis, the surgical area was evaluated, and no acute or chronic inflammatory process was observed. The aspect of proliferative tissue at bone-implant interface suggested that healing was insured without any inflammatory processes. All these aspects prove that the surgical technique was performed properly.

The bone-implant interface was covered in new bone matrix (Figure 1), but its thickness, proliferation and differentiation were different in various areas (Figures 2, 3). The newly formed tissue occupies the spaces between the implant's threads, yet its links to the adjacent structures varies. The thinnest new osseus tissue proliferation at bone-implant interface was found in the periosteal third of the experimental defect. Although it is presented as a typical bone structure, the new bone matrix is thin, with large gaps of fibrous and vascular tissue. In the contact area between the new proliferative bone tissue and the adjacent bone there was a layer of damaged bone with polymorphic cavities. This leads to the understanding that the binding of new osseus tissue to the adjacent tissues is still weak in this area. In the deeper layers, from region to region, the newly proliferated tissue may differ in thickness, as well as in its binding to the adjacent tissues. For the proliferative tissue, the endosteal area is thicker than the periosteal area and its mergings are different from one area to another.

At bone-implant interface, the newly proliferated bone tissue had a particular aspect, with an intermediate structure between cancellous and compact bones. The ratio of bone structure and trabecular structure had differences from one area to another. 


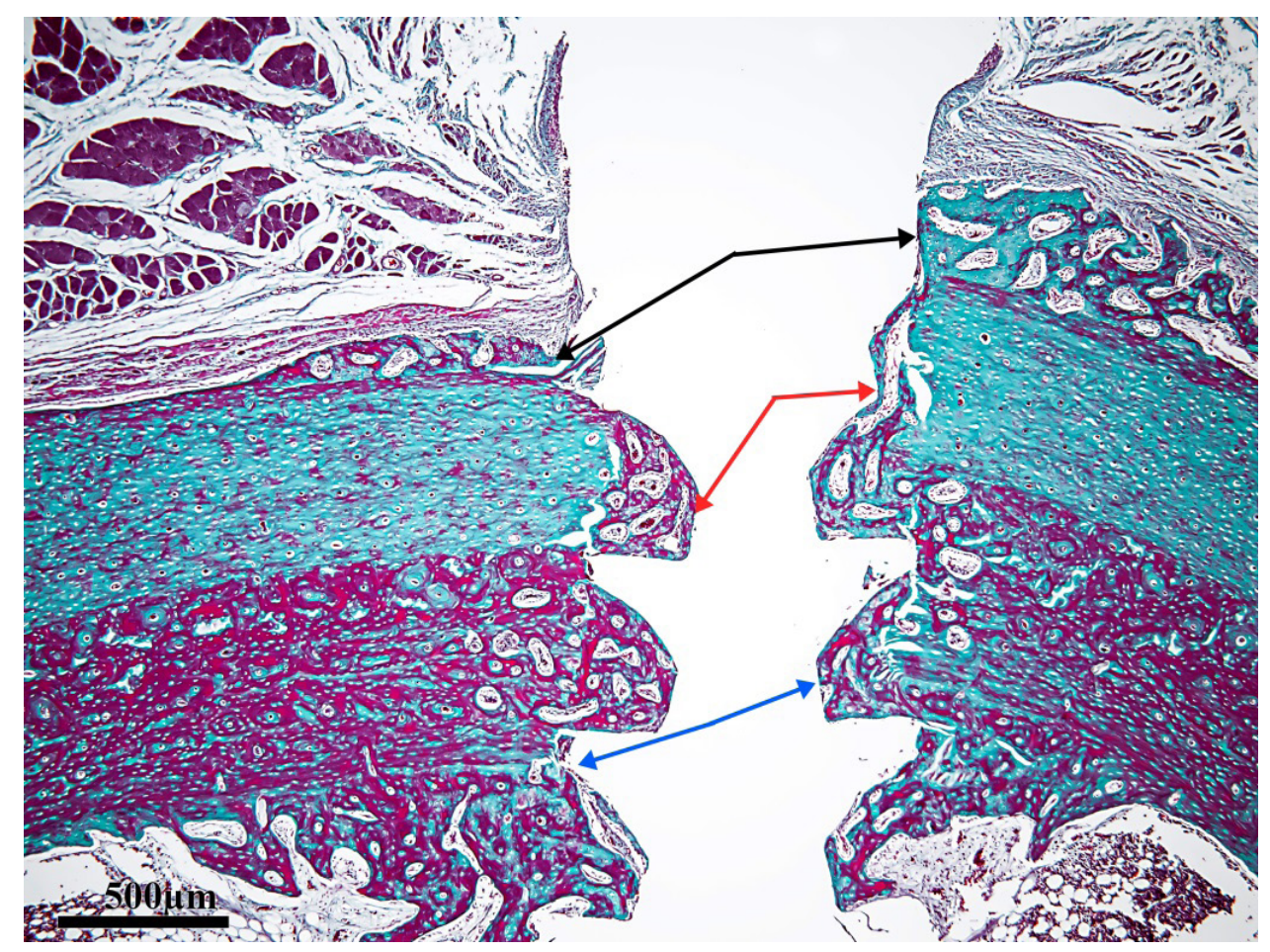

Figure1. 4 weeks after implant placement in males - bone/implant interface (TG), black arrow- periosteal area; blue arrow - endosteal area; red arrow - middle area.

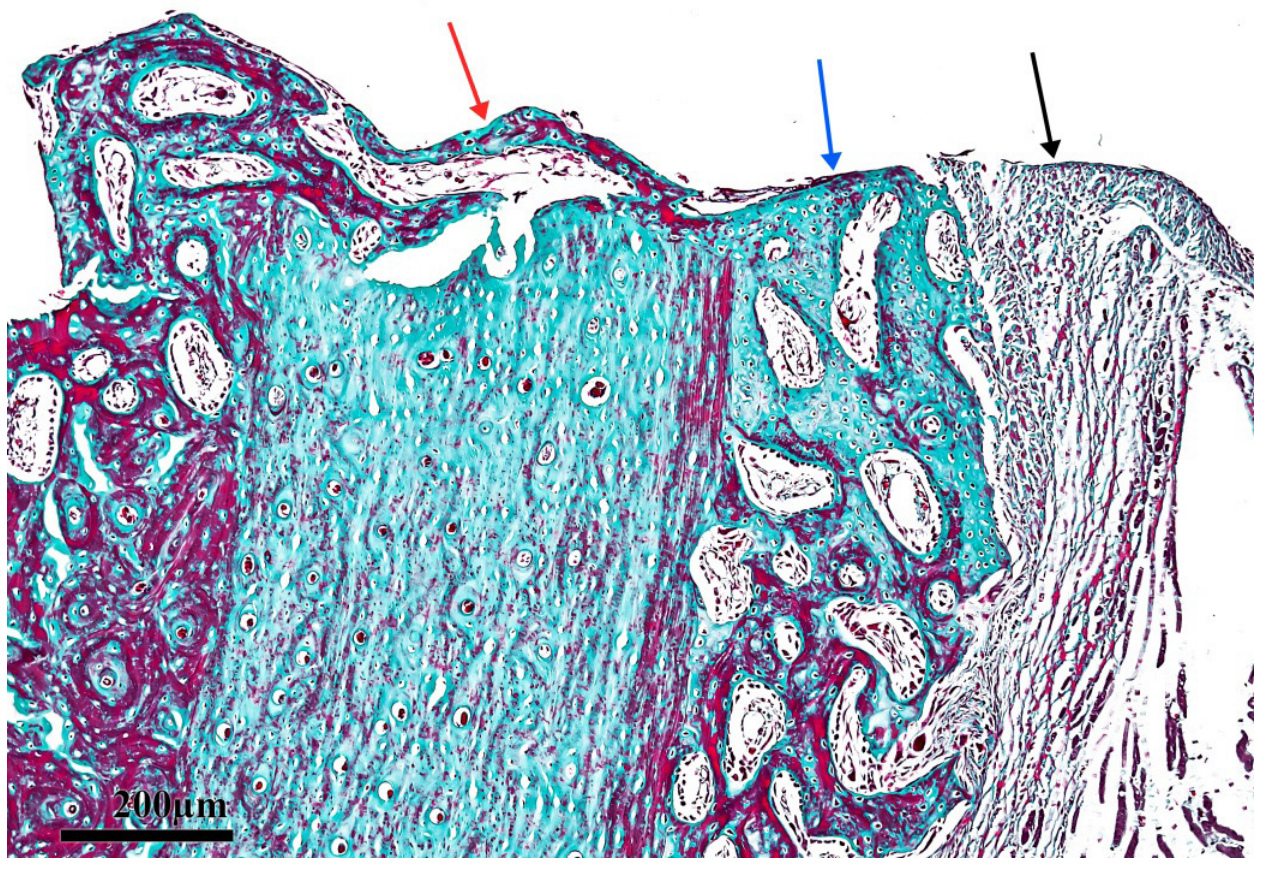

Figure 2. 4 weeks after implant placement in males - upper half of bone-implant interface (TG), black arrow - periosteum, blue arrow - periosteal area, red arrow bone trabeculae in the middle area. 


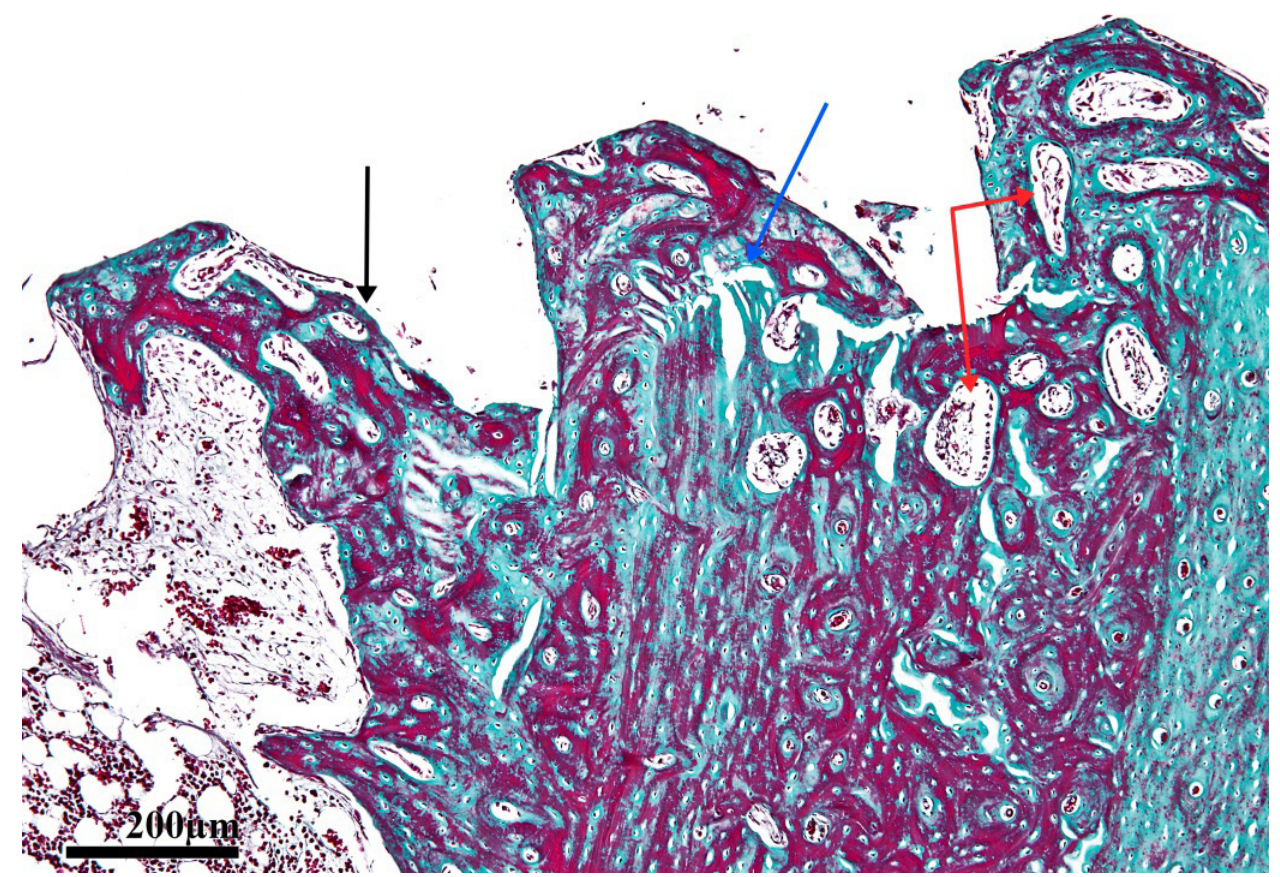

Figure 3. 4 weeks after implant placement in males - lower half of bone-implant interface (TG), black arrow - newly formed bone, endosteal area; blue arrow - damaged remaining bone; red arrow - areas of fibrous and vascular tissue.

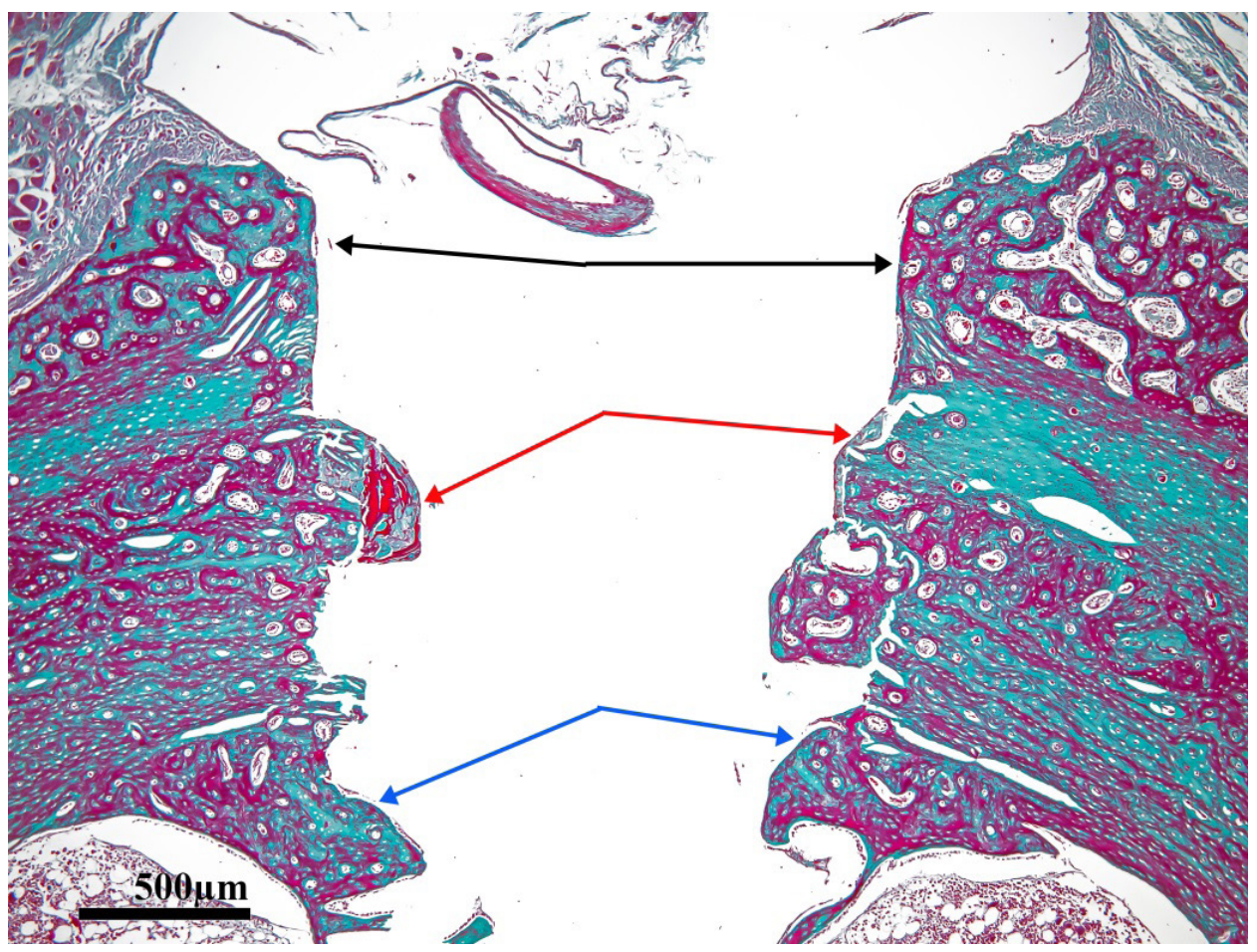

Figure 4. 4 weeks after implant placement in females - bone/implant interface (TG), black arrow- periosteal area; blue arrow - endosteal area; red arrow - middle area. 


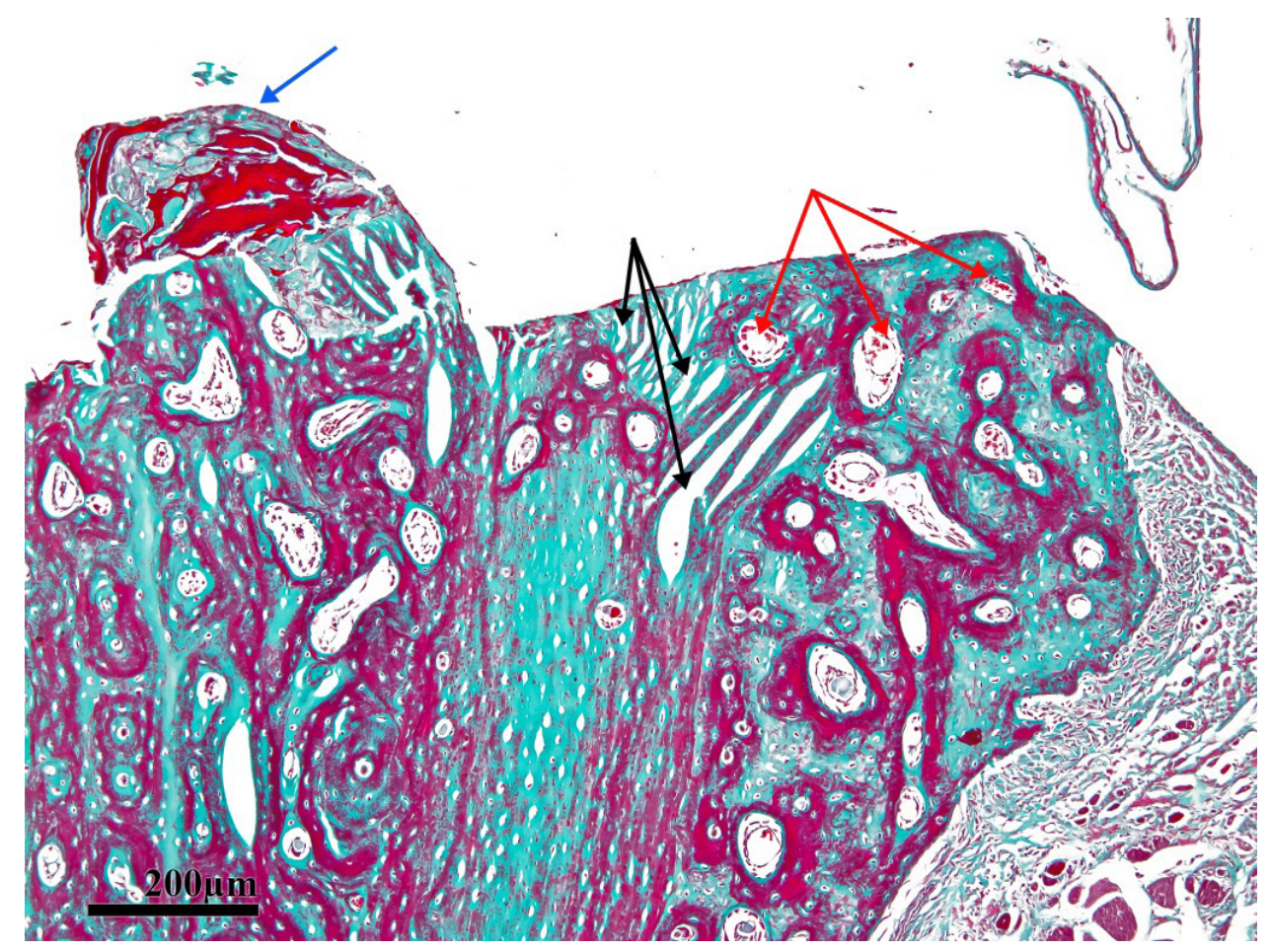

Figure 5. 4 weeks after implant placement in males - upper half of bone-implant interface (TG), black arrow - polymorphic cavities, blue arrow - augmentation material, red arrow - polymorphic areas of fibrous and vascular tissue.

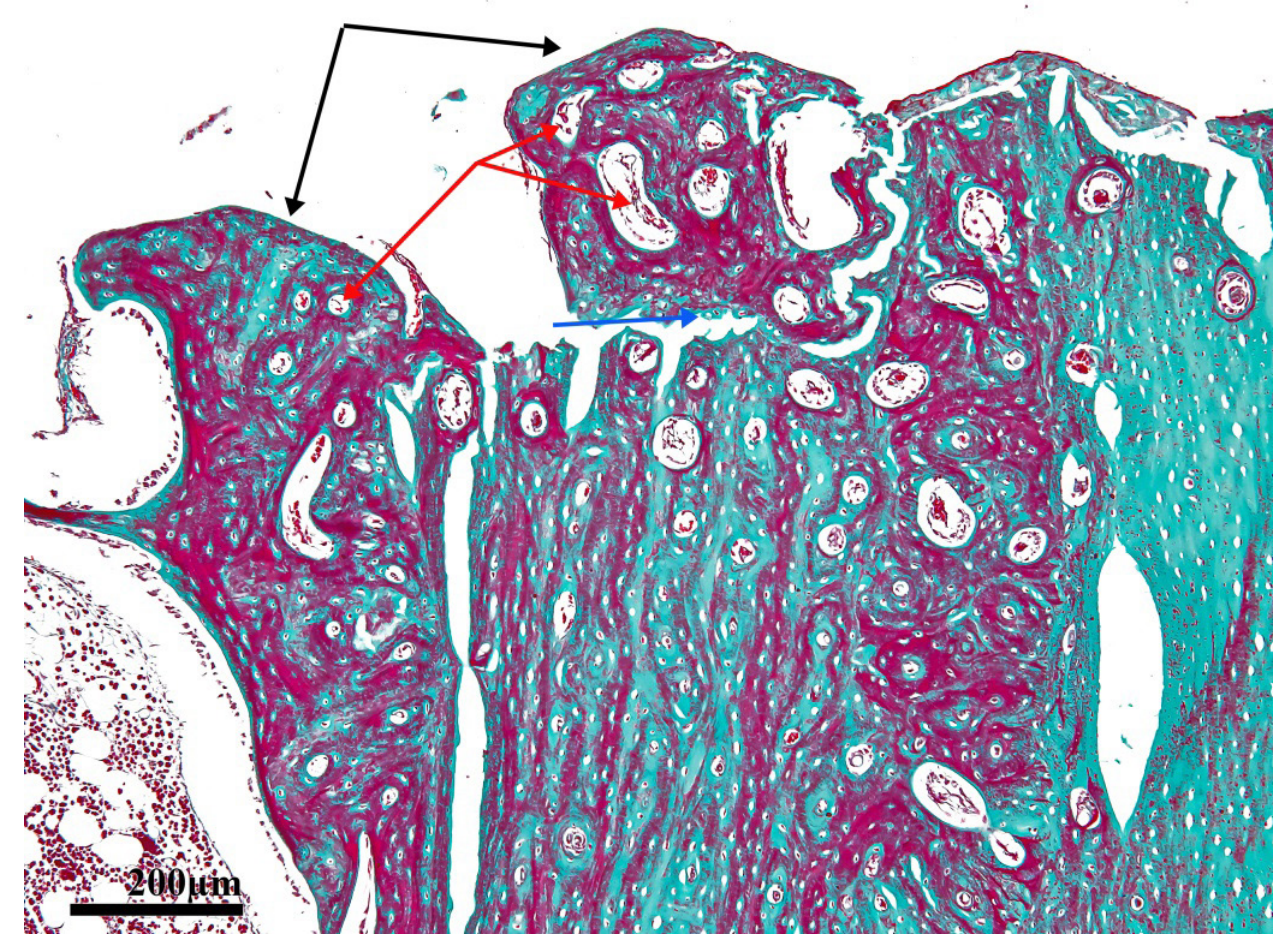

Figure 6. 4 weeks after implant placement in females - lower half of bone-implant interface (TG), black arrow - primary bone, blue arrow -junction area, and red arrow - polymorphic gaps. 
At the interface site, the proliferative osseus tissue was nonlamellar, young primary bone tissue, with a few scattered bone lamellae. This indicates bone remodelling to compact bone, though in an early stage.

In females, 4 weeks after implant placement, the surgical area was free of inflammatory processes in both soft and hard peri-implantation tissues. The latter structures were in close contact to the interface area, from the periosteal area to the endosteal one (Figure 4). In the periosteal third there was new proliferative osseus tissue arising from the periosteum. Furthermore, there was an area, near the interface of very thin, discontinuous newly proliferated tissue, and the deeper area was represented by damaged osseus tissue, with discontinuities. After that, the newly proliferated tissue was present and was found on $60-70 \%$ of the implant's threads and thread gaps, while the rest was occupied by augmentation material from the surgical self-tapping of the implants (Figure 5).

The endosteal third of the interface area presented new proliferated bone matrix, which fill the thread gaps between the threads (Figure 6).

Dental implant osteointegration and survival rate evaluation represent an area of interest for implantology specialists. Although there are various means used for this purpose, they do not provide the accurate information regarding specific dangerous factors that could affect the outcome. This whole process is influenced by a large number of factors that can affect different stages, alone or by a cumulative effect. Most studies have researched the implant survival rate, a result of cumulative factors that can affect this process. These were meta-analytical studies, such as the one conducted by Chrcanovic et al. in 2015, which states that dental implant placement in males has a higher long-term failure rate than in females. The various factors that can influence implant survival rate occur after the osteointegration process.

Early implant failure rate is influenced by a smaller number of risk factors than the implant survival rate, such as risk factors arising from the implant placement surgery and osteointegration process. Better understanding of early implant failure risk factors is of utmost importance for implantology specialists and their implant therapy management, adapted to every particular patient.

On patients, osteointegration process evaluation is possible only by non-invasive procedures, specifically by using imaging techniques. From a structural point of view, cellular processes that accompany the osteointegration processes, can be observed only by histological evaluation. Considering that this implies harvesting bone and implant, it cannot be performed on human patients; therefore experimental animal models have to be used. Although histological techniques are time consuming, the samples give excellent information about bone proliferation and healing (Rentsch et al., 2014). Results found in these studies serve as reference on osteointegration process evaluation, its stages, risk factors and waiting time necessary for peri-implantation bone proliferation, although it cannot be entirely extrapolated to humans.

For a detailed osteointegration process evaluation, quality histological sample processing was conducted for preserving peri-implantation soft tissues, as well as the hard tissue around the implantation bed. Following sample analysis, it was revealed that newly proliferated tissue at bone-implant interface is connected intimately to the implant surface, proliferated new tissue being found throughout the implant's threads. Newly proliferated bone tissue in contact with the implant surface attests to the qualities of the biomaterial, as well as to the proper healing of the experimental defect.

Comparative evaluation of osteointegration process took into consideration several aspects, such as the extent of newly proliferated bone tissue at bone-implant interface site, thickness and structure of said proliferated tissue and its alignment to the other bone structures. The extent of newly proliferated bone tissue in males is higher than in females, it covers the whole bone-implant interface in a continuous layer, and it covers all the implant's threads and thread gaps. Meanwhile in females there is a central gap, there are certain threads and thread gaps left uncovered.

The thickness of the proliferative osseus tissue varies. It is the thickest in the endosteal area, followed by the periosteal area and the middle area in both males and females. If newly proliferated bone tissue thickness is compared between genders, there is a significantly thicker layer in males than females.

The newly proliferated bone tissue is a primary bone in different stages of remodelling and it is similar to cancellous bone. In males there are fewer and smaller cone cavities than in females, 
thus newly proliferated tissue density and resistance at interface area at 4 weeks is higher in males than females.

At interface site, the binding of newly proliferated bone tissue to its surrounding tissues is different from an area to another, as well as between genders. The best merger was found in the endosteal area, followed by the periosteal area and the middle one, although incomplete in all areas, making it still vulnerable at 4 weeks after implant placement. Comparing the situation between genders, the tissue connections are more advanced in males than females.

Overall osteointegration process, at 4 weeks after implant placement, evolved well, although it was far from over. The osteointegration process follows certain stages, which were similar between genders, but there was a difference regarding the speed of progression through certain stages. Thus, the osteointegration process at 4 weeks after implant placement was $25 \%$ more advanced in males. This aspect is clinically relevant for the appropriate evaluation of waiting periods from implant placement to prosthetic mounting, which should be longer in females.

\section{Conclusions}

The newly proliferated tissue at bone-implant interface was represented in all animals by bone tissue in close contact with the implant surface, which attest to the qualities of this biomaterial. The osteointegration process follows the same stages in all rabbits, but the speed of progression is $25 \%$ higher in males. The higher progression rate through stages, in males, must be taken into consideration for the appropriate evaluation of waiting periods, from implant placement to prosthetic mounting.

Acknowledgments. This research did not receive any specific grant from funding agencies in the public, commercial, or not-for-profit sectors.

\section{References}

1. Akca K, Akkocaoglu M, Comert A, Tekdemir I, Cehreli MC (2007). Bone strains around immediately loaded implants supporting mandibular overdentures in human cadavers. Int. J. Oral Maxillofac. Implants., 22:101-109.

2. Bancroft JD, Layton C (2019). Connective and other mesenchymal tissues with their stainsin Bancroft's Theory and Practice of Histological Techniques (Eighth Edition) , p. 153-175

3. Chrcanovic BR, Albrektsson T, Wennerberg A (2015). Dental implants inserted in male versus female patients: a systematic review and meta-analysis, Journal of Oral Rehabilitation 42; 709-722

4. Gabe M (1968). Techniques Histologiques, Masson Et C $\mathrm{C}^{\mathrm{ie}}$, Editeurs, Paris

5. Gruber HE (1992). Adaptations of Goldner's Masson trichrome stain for the study of undecalcified plastic embedded bone. Biotech Histochem 67:30-34,

6. Gruber HE, Ingram JA (2003). Basic Staining and Histochemical Techniques and Immunohistochemical Localizations Using Bone Sections In: Handbook of Histology Methods for Bone and Cartilage Edited by:Y. H. An and K. L. Martin (C) Humana Press Inc., Totowa, p.181

7. Haas R, Mensdorff-Pouilly N, Mailath G, Watzek G (1996). Survival of 1,920 IMZ implants followed for up to 100 months. Int. J. Oral Maxillofac Implants 11:581-588

8. Mesa F, Munoz R, Noguerol B, de Dios Luna J, Galindo P (2008). Multivariate study of factors influencing primary dental implant stability. Clin. Oral. Implants. Res., 19:196200.

9. O"stman PO, Hellman M, Wendelhag I, Sennerby L (2006). Resonance frequency analysis measurements of implants at placement surgery. The International Journal of Prosthodontics 19:77-83.

10. Pearce AI, Richards RG, Milz S, Schneider E, Pearce SG (2007). Animal models for implant biomaterial research in bone: a review. Eur Cell Mater; 13:1 - 10;

11. Raghavendra S, Wood MC, Taylor TD (2005). Early wound healing around endosseous implants: a review of the literature. International Journal of Oral \& Maxillofacial Implants 20: 425-431

12. Rentsch C, Schneiders W, Manthey S, Rentsch B, Rammelt S (2014). Comprehensive histological evaluation of bone implants, Biomatter, 4:1, e27993, DOI: 10.4161/ biom. 27993

13. Tricio J, Laohapand P, van Steenberghe D, Quirynen M, Naert I (1995). Mechanical state assessment of the implant-bone continuum: a better understanding of the Periotest method. International Journal of Oral \& Maxillofacial Implants 10: 43-49.

14. Wancket L M (2015). Animal Models for Evaluation of Bone Implants and Devices: Comparative Bone Structure and Common Model Use, Veterinary Pathology 52(5), p. 842-850 